\title{
DUAS ESPÉCIES NOVAS DE LOPHOBLATTA (BLATTARIA, BLATTELLIDAE) DO BRASIL E DESCRIÇÃO DA GENITÁLIA DE CINCO ESPÉCIES CONHECIDAS
}

\author{
Sonia Maria Lopes ${ }^{1,2}$ \\ Edivar Heeren de Oliveira ${ }^{1}$
}

\begin{abstract}
TWO NEW SPECIES OF LOPHOBLATTA (BLATTARIA, BLATTELLIDAE) FROM BRAZIL AND DESCRIPTION OF GENITALIA OF FIVE KNOWN SPECIES. New species described: Lophoblatta bromelicola and L. tingua both from Rio de Janeiro, Brazil. The male genitalia of five other species are illustrated and described. New records are given: L. petropolitana Rocha e Silva \& Vasconcelos, 1987 to Espírito Santo and L. speerae Rocha e Silva-Albuquerque \& Gurney, 1963 to Pernambuco, Goiás and Espírito Santo, Brazil. The genus is divided in four groups according as the male genitalia characters.
\end{abstract}

KEYWORDS. Lophoblatta, taxonomy, new species, Neotropical.

\section{INTRODUÇÃO}

O gênero neotropical Lophoblatta Hebard, 1929 foi proposto com base em Blatta pellucida Burmeister, 1838 e comparado a Neoblattella Shelford, 1911 e a Latiblattella Hebard, 1919. HeBARD (1929) utilizou caracteres como a largura do pronoto e tipo de modificação nos tergitos abdominais para diferenciá-lo dos gêneros citados. Oito espécies são conhecidas e associadas às coletas no solo e em bromélias: $L$. arawaka Hebard, 1929, L. arlei Rocha e Silva-Albuquerque, 1964, L. brevis Rehn, 1937, L. fissa (Saussure \& Zehntner, 1893), L. fuliginosa Princis, 1965, L. pellucida (Burmeister, 1838), L. petropolitana Rocha e Silva \& Vasconcelos, 1987 e L. speerae Rocha e Silva-Albuquerque \& Gurney, 1963. Acrescentam-se duas espécies novas ao gênero e descreve-se a genitália do macho de cinco espécies descritas apenas por caracteres morfológicos de pernas, asas e coloração; registra-se pela primeira vez a ocorrência de L. petropolitana R. e Silva \& Vasconcelos, 1987 para o Espírito Santo e L. speerae Rocha e Silva-Albuquerque \& Gurney, 1963 para Pernambuco, Goiás e Espírito Santo, Brasil e divide-se o gênero em quatro grupos de acordo com caracteres da genitália dos machos. A distribuição

\footnotetext{
1. Depto de Entomologia, Museu Nacional, Universidade Federal do Rio de Janeiro, Quinta da Boa Vista, São Cristóvão, 20940-040, RJ, Brasil. (sonialf@acd.ufrj.br).

2. Programa de Pós-Graduação (Doutorado) em Biologia Animal, Universidade Federal Rural do Rio de Janeiro, BR 465 Km 7 , Seropédica, 23890-000, RJ, Brasil.
} 
geográfica do gênero na América do Sul estende-se da Venezuela até o sul do Brasil e em alguns países da América Central.

A genitália dos machos foi analisada, utilizando as técnicas descritas em LoPEs \& Oliveira (2000b). A terminologia seguiu McKiтtrick (1964). O material estudado está depositado na coleção do Museu Nacional, Universidade Federal do Rio de Janeiro (MNRJ).

\section{Lophoblatta Hebard, 1929}

Lophoblatta Hebard, 1929:350; Rehn, 1949:12; Princis \& Kevan, 1955:164; Princis, 1955:8; Bruinning, 1959:58; R. e S.-Albuquerque \& Gurney, 1963:59; R. e S.-Albuquerrque, 1964c:1; McKittrick, 1964:17; Rотн, 1968:99.

Espécie-tipo. Blatta pellucida Burmeister, 1838.

\section{Lophoblatta bromelicola sp. nov.}

(Figs. 1-11)

ॐ. Colorido geral castanho-claro uniforme. Olhos e espaço interocular com faixa transversal castanho-escura. Fronte com manchas castanho-escuras entre e abaixo da base das inserções das antenas (fig. 1); palpos maxilares com o último artículo escurecido. Pronoto uniformemente brilhante (fig. 2). Pernas delineadas na base das coxas e na base dos espinhos dos fêmures de castanho-escuro. Pulvilos esbranquiçados.

Cabeça com vértice exposto. Espaço interocular amplo e semelhante ao que separa a base das inserções antenais. Antenas longas, pilosas e filiformes, ultrapassando o ápice dos cercos. Palpos maxilares com o terceiro artículo levemente maior que o quarto; quinto artículo dilatado, piloso e menor que os anteriores. Pronoto convexo, trapezoidal de ápice reto, laterais suavemente arredondadas e base levemente angular (fig. 2).

Tórax com tégminas desenvolvidas que ultrapassam o ápice dos cercos. Campo marginal côncavo e marcado. Campo escapular reto próximo à base do campo discoidal, este curvo no ápice da tégmina, seguindo reto até o campo anal que é amplo e convexo. Fêmur I com disposição de espinhos tipo A3; face póstero-ventral com cinco espinhos grandes, sendo um deles apical. Fêmures II e III com espinhos semelhantes, grandes e espaçados nas faces ântero- e póstero-ventrais. Pulvilos presentes. Arólios desenvolvidos. Unhas tarsais simétricas e serrilhadas internamente.

Abdome com modificação tergal nos sétimo e oitavo segmentos, com cílios agrupados medianamente no sétimo e dispersos no oitavo (fig. 3). Placa supra-anal projetada entre os cercos, com ápice reto e cílios esparsos. Paraproctos bem evidenciados, com cílios apicais. Cercos pilosos, muito desenvolvidos, afilando para o ápice (fig. 4). Placa subgenital larga; estilos simples com cílios nos ápices (fig. 8). L2vm bífido. L2d apicalmente com projeções espiniformes curtas e robustas dispostas por toda a superfície, sendo duas maiores; ramo lateral apicalmente com várias projeções espiniformes (fig. 10). L1 com os braços assimétricos e alargados: um desenvolvido e outro reduzido. Estrutura mediana alargada e disforme, apicalmente com vários espinhos de porte médio, robustos e ciliforme látero-apicalmente (fig. 9). R2 sinuoso, cuja extremidade termina em forma de bico de ave (fig. 11). Conjunto de escleritos do R2 de configuração variada: um deles como um penacho, outro espiniforme e o último digitiforme, coberto de cílios (figs. 5-7). 

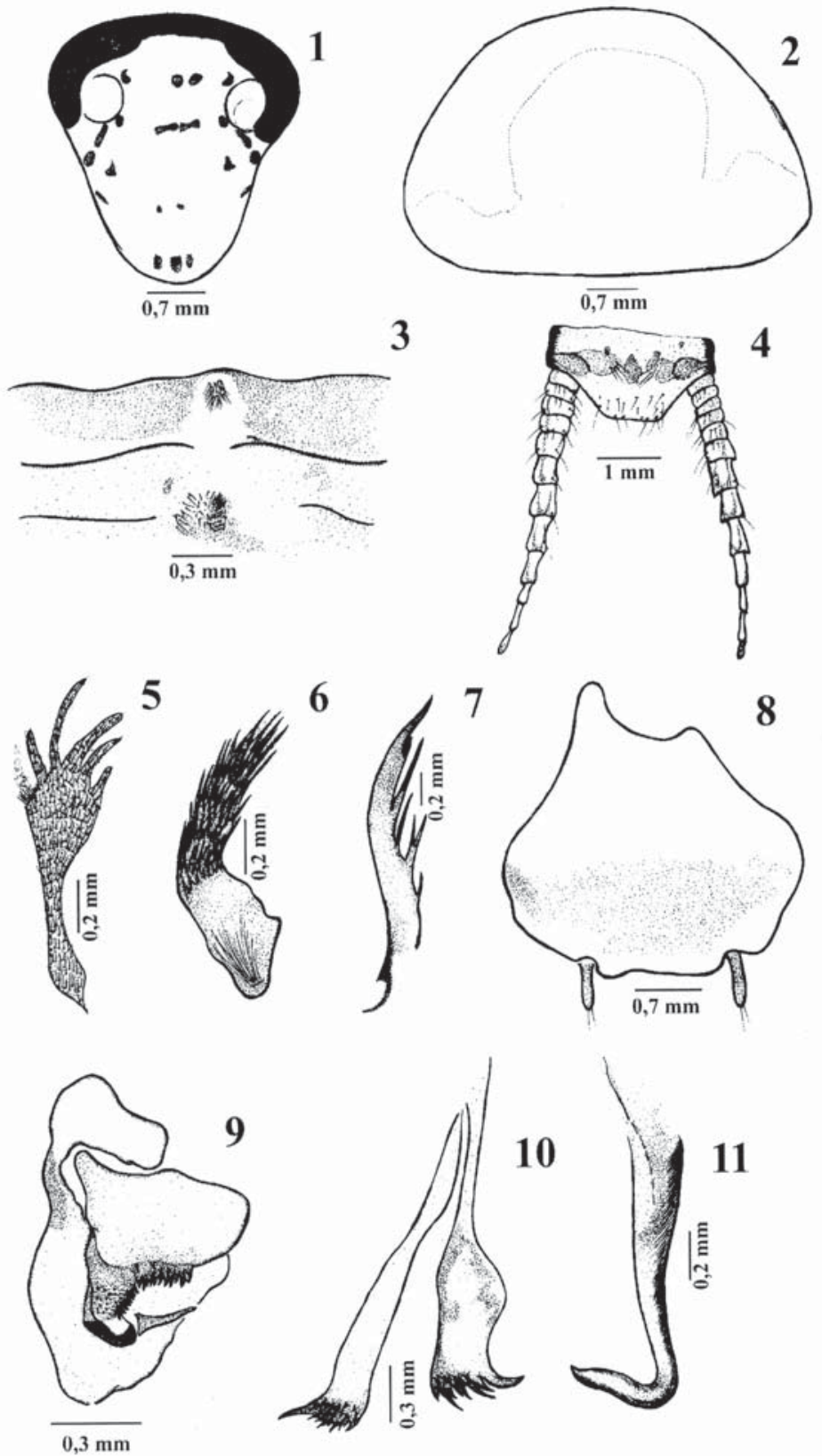

Figs. 1-11. Lophoblatta bromelicola sp. nov., holótipo o. 1, cabeça, ventral; 2, pronoto, dorsal; 3, modificação tergal do abdome, dorsal; 4, placa supra-anal, dorsal; 5-7, escleritos de R2; 8, placa subgenital, ventral; 9, L1; 10, L2vm; 11, R2. 
Dimensões em mm. Comprimento total 17,0; comprimento do pronoto 4,5, largura 6,5; comprimento da tégmina 14,5, largura 5,0.

Material-tipo. Holótipo đ’, BRASIL, Rio de Janeiro: Jacarepaguá, VII.1972, P.S. Abreu col., em bromélia (MNRJ).

Etimologia. O espécime foi coletado em bromélia, que deu origem ao nome específico.

Diagnose. A espécie distingue-se das demais pela ausência de coloração no pronoto, pela configuração da modificação tergal no abdome e do L2vm na genitália do macho, como descrito anteriormente.

\section{Lophoblatta tingua sp. nov.}

(Figs. 12-24)

ð. Colorido geral castanho-claro brilhante. Faixa transversal no espaço interocular (fig. 12), último artículo dos palpos maxilares, coxas e bases dos espinhos dos fêmures castanho-escuros. Pronoto com a maior parte do disco central transparente e brilhante, deixando visíveis marcações internas pouco notadas (fig. 13). Tégminas com o tronco inicial de todas as nervuras levemente mais escuro.

Cabeça com espaço interocular amplo e semelhante ao que separa as bases das inserções antenais. Antenas longas, pilosas e filiformes, ultrapassando o ápice dos cercos. Palpos maxilares com o $3^{\circ}$ artículo levemente maior que o $4^{\circ} ; 5^{\circ}$ artículo dilatado, piloso e menor que os dois anteriores. Pronoto convexo trapezoidal de ápice reto, laterais suavemente arredondadas e base levemente angular.

Tórax com tégminas bem desenvolvidas, ultrapassando o ápice dos cercos. Campo marginal côncavo e marcado. Campo escapular reto próximo à base do campo discoidal, este curvo no ápice da tégmina, seguindo reto até o campo anal, que é amplo e convexo. Fêmur I com disposição de espinhos tipo A3; face póstero-ventral com cinco espinhos grandes, um deles apical. Fêmures II e III com espinhos semelhantes; espinhos grandes e espaçados nas faces ântero- e póstero-ventrais. Pulvilos presentes e arólios desenvolvidos; unhas tarsais simétricas e serrilhadas internamente.

Abdome com modificação tergal mediana no sétimo segmento, com várias séries de cílios dispostas em diferentes níveis (fig. 15). Placa supra-anal projetada medianamente entre os cercos, com cílios finos e longos. Paraproctos evidenciados com cílios apicais (fig. 14). Placa subgenital assimétrica ciliada; estilos pouco diferenciados e ciliados apicalmente (fig. 17). L2vm bífido. L2d alargado com espinhos curtos e robustos por toda superfície apical, sendo dois maiores nas extremidades opostas (figs. 20, 23); ramo lateral com projeções digitiformes no ápice (figs. 20, 22). L1 com os braços assimétricos: um desenvolvido e afilado e outro curto, de ápice arredondado. Estrutura mediana alargada e disforme com vários espinhos pequenos e robustos (fig. 18). Escleritos do R2 semelhantes a ganchos espiniformes (figs. 16, 19, 21). R3 com uma das extremidades mais alargada (fig. 24).

Dimensões em mm. Comprimento total 16,0; comprimento do pronoto 4,0, largura 6,0; comprimento da tégmina 12,0, largura 4,0.

Material-tipo. Holótipo $ð$, BRASIL, Rio de Janeiro: Jacarepaguá, Reserva do Tinguá,10.VIII.1973, N. Santos, Machado Filho e A. Castro col., em bromélia (MNRJ). 


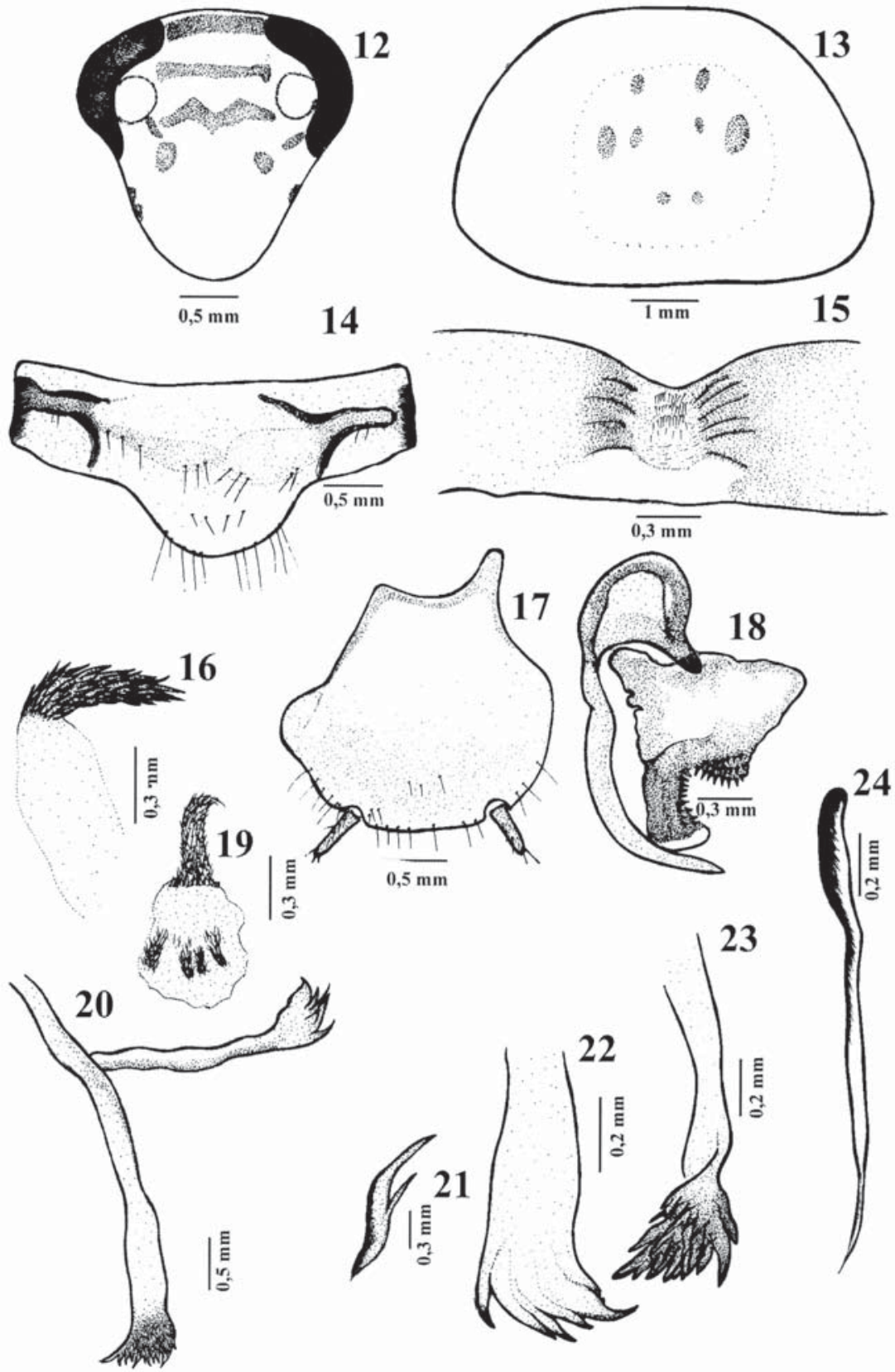

Figs. 12-24. Lophoblatta tingua sp. nov., holótipo o. 12, cabeça, ventral; 13, pronoto, dorsal; 14, placa supra-anal, dorsal; 15, modificação tergal do abdome, dorsal; 16, 19, 21, escleritos de R2; 17, placa subgenital, ventral; 18, L1; 20, 22, 23, L2vm; 24, R3. 
Etimologia. Substantivo em aposição alusivo à localidade de coleta.

Diagnose. A espécie é distinta entre as demais pela configuração da modificação tergal no abdome e do L2vm na genitália do macho, descrito anteriormente.

\section{Lophoblatta arlei Rocha e Silva-Albuquerque, 1964}

(Figs. 25-33)

Lophoblatta arlei R. e S.-Albuquerque, 1964c:2; Roth, 1968:105; R. E S.-Albuquerque, 1972:26; Lopes \& Oliveira, 2000a:20.

Localidade-tipo. Holótipo ơ, Brasil (Pará) (MNRJ).

Distribuição. Peru e Brasil (Amazonas, Pará).

ऊ. Abdome com modificação tergal do sétimo segmento, na região mediana, em forma de tufo simétrico de cílios, que decresce em uma fileira igualmente simétrica (fig. 26). Placas supra-anal e subgenital (figs. 25, 28) descritas em R. E S.-AlbuQuerque (1964b). L2vm bífido, L2d bifurcado com as extremidades acuminadas em forma de unha. Ramo lateral com o ápice bifurcado: uma extremidade terminada em espinho e a outra arredondada, com uma projeção espiniforme diminuta (figs. 32, 33). L1 com os braços assimétricos, um deles afilado, muito desenvolvido e o outro pouco perceptível; estrutura mediana linguiforme com duas expansões que se sobrepõem a ela, terminadas por formações espinhosas (fig. 27). R2 em forma de gancho com o ápice terminando em espinho diminuto (fig. 29). Escleritos do R2 em forma de pente com os dentes espaçados (fig. 30). R3 afilado em uma das extremidades e espatulado na outra (fig. 31).

Material examinado. BRASIL, Pará: Boa Vista, o, I.1965, França col. (dissecado); Castanhal †, II.1964, Arlé col.; Belém I.A.N., †, IX.1965, W. França col.; Utinga, Ơ', II.1965, Arlé col.; Ananindeua, Guajará, ơ, IX.1964, P. Waldir \& Souza col.

\section{Lophoblatta brevis Rehn, 1937}

(Figs. 34-41)

Lophoblatta brevis Rehn, 1937:197; Bruijning, 1959:60; R. E S.-Albuquerque, 1964b:12; 1964c:4; Roth, 1968:99; R. e Silva, 1982:14.

Localidade-tipo. Holótipo đ’, Guiana (Upper Essequibo River) (The Natural History Museum).

Distribuição. Guiana, Suriname, Venezuela, Equador e Brasil (Amazonas, Rio de Janeiro).

Ђ. Abdome com modificação tergal nos sétimo e oitavo segmentos, com um tufo de pêlos simétricos medianamente (fig. 35). Placa supra-anal pilosa, projetada entre os cercos, com pequena reentrância mediana. Cercos alargados e curtos (fig. 34). Placa subgenital larga, com reentrância mediana entre os estilos e cílios nas áreas protuberantes; estilos simples, pilosos (fig. 36). L2vm bífido, alongado; L2d com três espinhos diminutos e um prolongamento lateral pré-apical; ramo lateral alargado no ápice e flamuriforme (figs. 40, 41). L1 com os braços assimétricos, um deles desenvolvido, embora afilado e o outro quase ausente; estrutura mediana alargada e disforme, com esclerotinização mediana (fig. 38). R2 em forma de gancho, com a extremidade afilada, terminando em espinho (fig. 37). R3 muito afilado em uma das extremidades e espatulado na outra (fig. 39).

Material examinado. BRASIL, Rio de Janeiro: Pedro do Rio, \&, 8.I.1934, Souto Maior col.; EQUADOR, Pastaza: Puyo, 900m, 2 ऊ, f, 14.X.1937, Clarke, Macintyre, Brundege col. (dissecado) (MNRJ). 


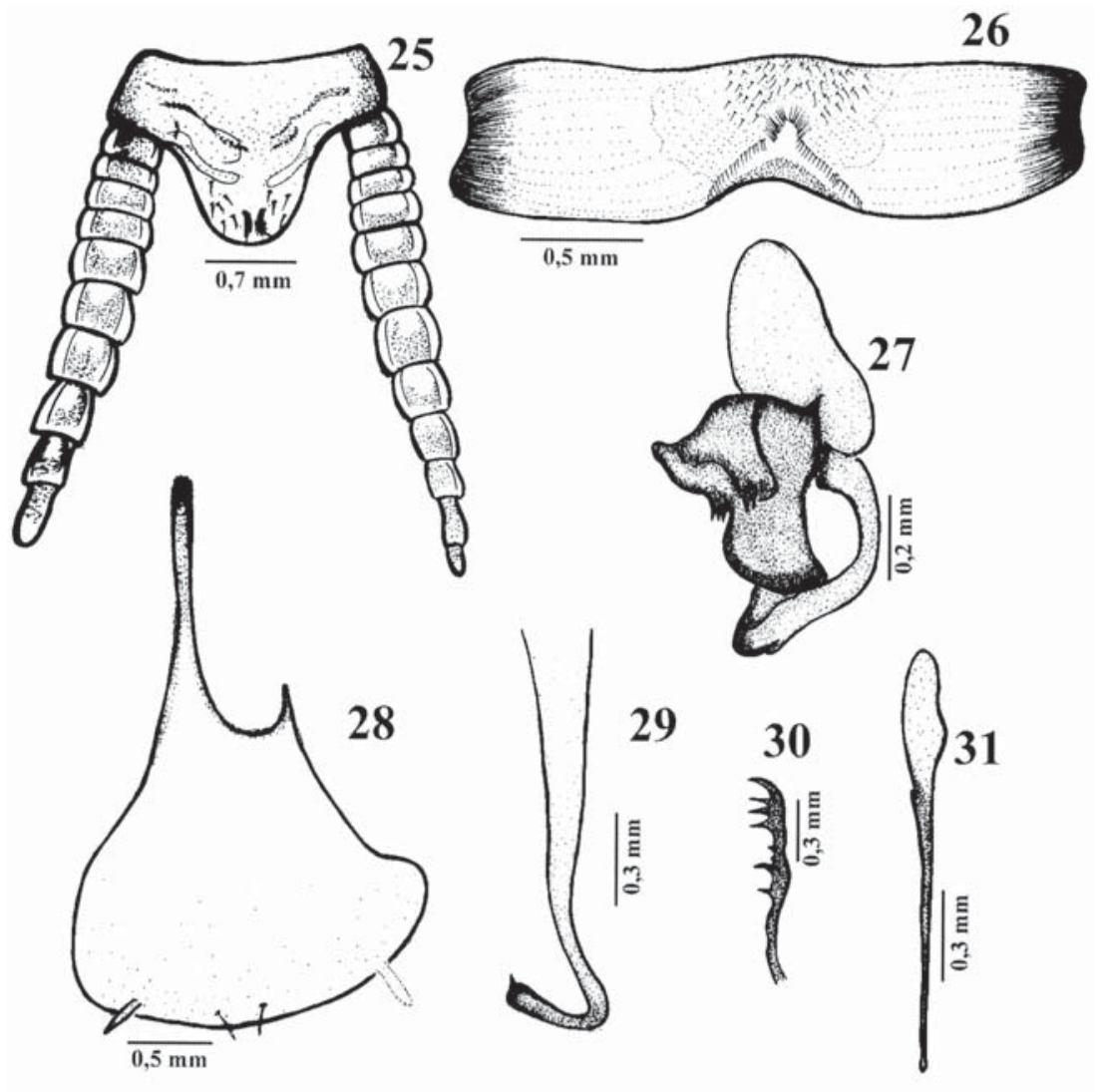

32
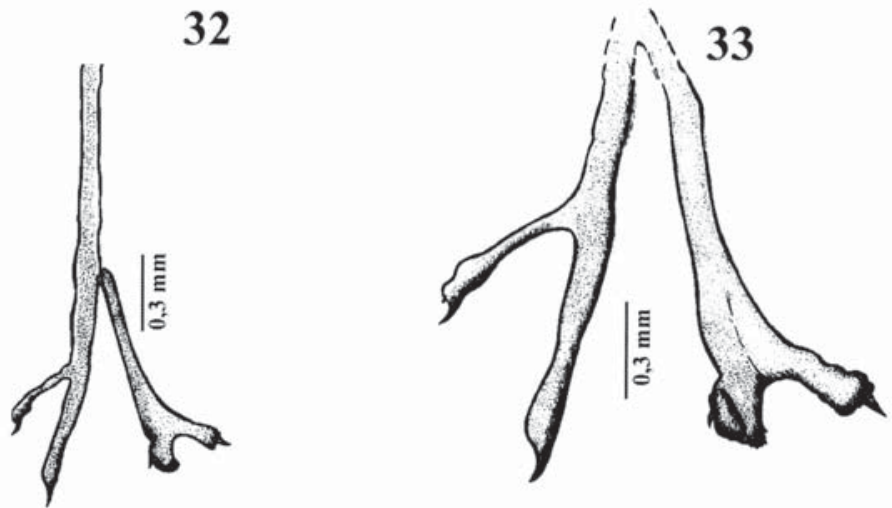

Figs. 25-33. Lophoblatta arlei R. e S.-Albuquerque, 1964, ơ. 25, placa supra-anal, dorsal; 26, modificação tergal do abdome, dorsal; 27, L1; 28, placa subgenital, ventral; 29, R2; 30, esclerito do R2; 31, R3; 32, 33, L2vm. 

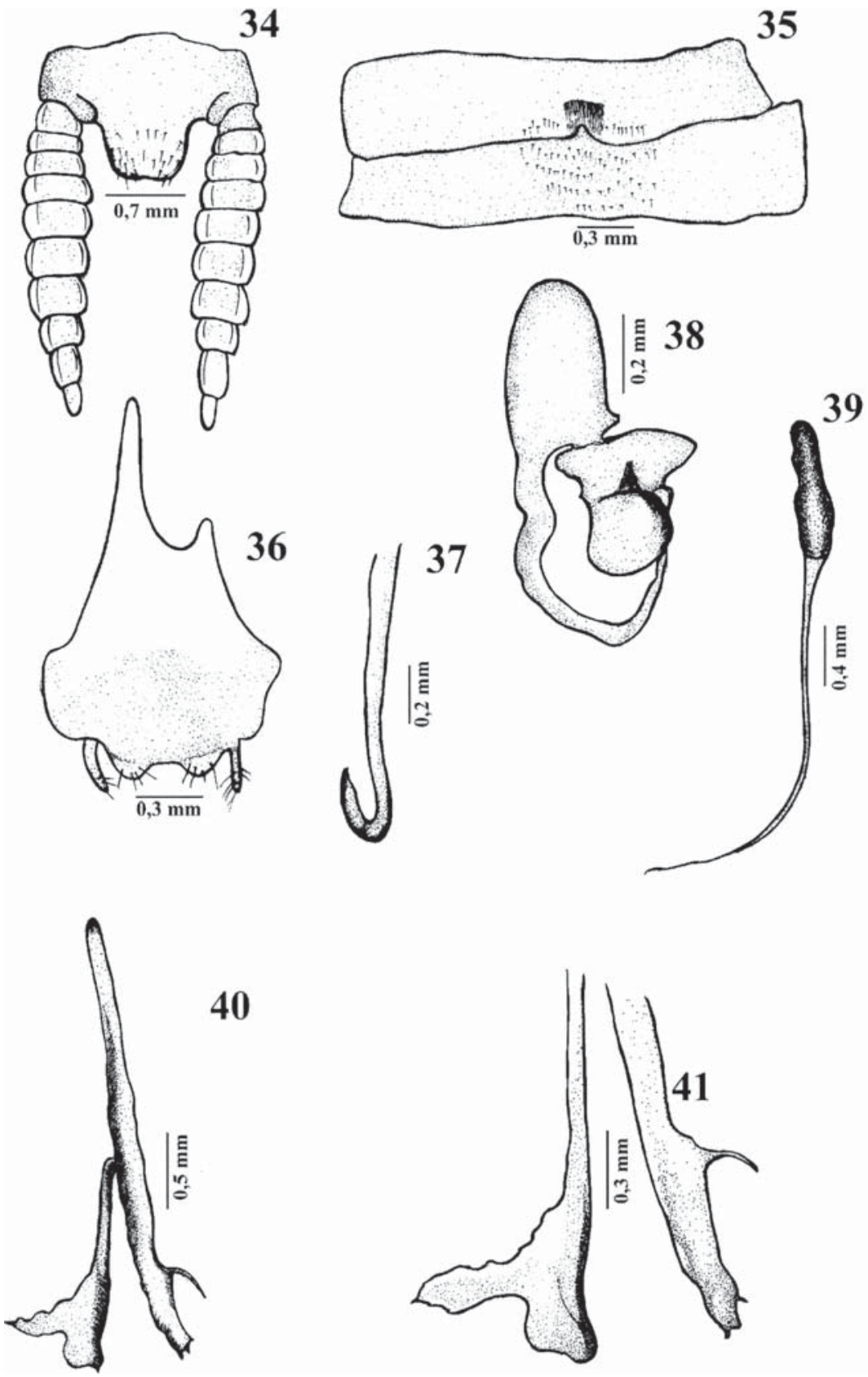

Figs. 34-41. Lophoblatta brevis Rehn, 1937, O’. 34, placa supra-anal, dorsal; 35, modificação tergal do abdome, dorsal; 36, placa subgenital, ventral; 37, R2; 38, L1; 39, R3; 40, 41, L2vm. 


\section{Lophoblatta pellucida (Burmeister, 1838)}

(Figs. 42-49)

Blatta pellucida BurmeIsTer, 1838:498; BRUNNER, 1865:109; KirBY, 1904:95.

Phyllodromia pellucida; SHELFORD, 1908:14.

Neoblattella pellucida; ReHn, 1916:230; 1918:157; HeBARD, 1926:163.

Lophoblatta pellucida; Hebard, 1929:350; Rehn, 1937:197-203; Bruijning, 1959:59; R. E S.-Albuquerque, 1964a:26; 1964c:4; 1972:26.

Localidade-tipo. Holótipo ơ, Brasil (Pará) (Academy of Natural Sciences, Philadelphia).

Distribuição. Guiana Francesa, Suriname e Brasil (Amazonas, Amapá, Pará, Paraíba, Mato Grosso).

o. Abdome com modificação tergal no oitavo segmento reduzida, sendo o mesmo côncavo medianamente, apresentando logo abaixo e próximo a essa concavidade cílios dispersos (fig. 43). Placas supra-anal e subgenital (figs. 42, 44) descritas em R. E S.AlbuQuerque (1964a). L2vm bífido; L2d próximo ao ápice, com um espinho diminuto seguido de uma projeção espiniforme; ramo lateral apicalmente com duas protuberâncias, uma alargada e a outra bastante afilada (figs. 48, 49). L1 com os braços assimétricos alargados: um desenvolvido, apicalmente em forma laminar e outro menor (fig. 45). R2 em forma de gancho, apresentando no ápice um pequeno espinho; região médio-basal alargada (fig. 46). R3 longo, com uma das extremidades afilada e a outra espatulada (fig. 47).

Material examinado. BRASIL, Pará: Belém (I.A.N.), 3 \&, 16.IV.1963, Werner col.; Utinga, ơ, II.1965, Waldyr col.; Boa Vista, ơ, I.1965, França col. (dissecado); 2 హ', f, I.64, Arlé col.; Castanhal (Pirelli), ક, V.1964, Souza col.; Igarapé-Assu (Fazenda Bonsucesso), †, IX.1964, Souza \& Waldir col.; Peixe-Boi, 2 \&, 20.II.1964, Arlé col.; Vigia, \&, III.1964, Arlé col.; Mato Grosso: Vera, đ', X.1973, Alvarenga \& Roppa col.

\section{Lophoblatta petropolitana Rocha e Silva \& Vasconcelos, 1987}

(Figs. 50-60)

Lophoblatta petropolitana R. E Silva \& Vasconcelos, 1987:6; Lopes \& Oliveira, 2000a:20

Localidade-tipo. Holótipo ơ, Brasil (Rio de Janeiro) (MNRJ).

Distribuição. Brasil (Rio de Janeiro, Espírito Santo, nova ocorrência.

§. Abdome com modificação tergal no sétimo segmento, medianamente, em forma de um grupo de cílios (fig. 51). Placas supra-anal e subgenital (figs. 50, 52), descritas em R. E SILVA \& VASCONCELOS (1987). L2vm bífido. L2d apicalmente terminando em projeções espiniformes dispostas na superfície superior; ramo lateral alongado cujo ápice apresenta projeções espiniformes (figs. 59, 60). L1 com os braços assimétricos: um deles desenvolvido, de ápice curvo e alargado, o outro menor. Estrutura mediana alargada esclerotinizada, com espinhos dispostos na superfície látero-apical (fig. 57). R2 em forma de gancho levemente dilatado próximo ao ápice curvo e acuminado (fig. 58). Conjunto de escleritos do R2, um deles em forma de mão coberta de cílios, os outros dois com ápices bem afilados (figs. 54-56). R3 longo com uma das extremidades afilada e a outra espatulada (fig. 53).

Material examinado. BRASIL, Rio de Janeiro: Grumari, $\subsetneq$ e 1 exemplar sem abdome, 3.VII.1975 (em bromélia); Macaé, 3 đ, 6 (dissecado); Espírito Santo: Vitória (Morro Moscoso), 3 ॐ, f, III.1979 (MNRJ). 

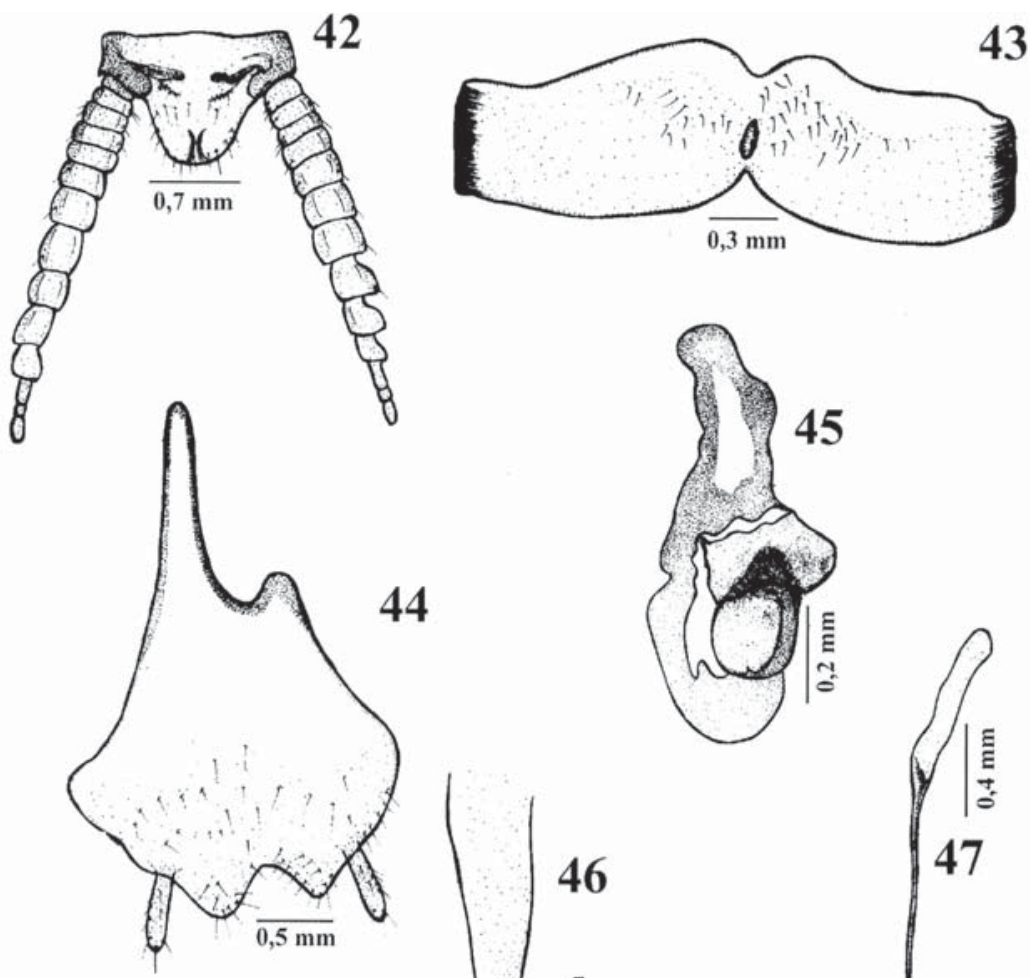

44
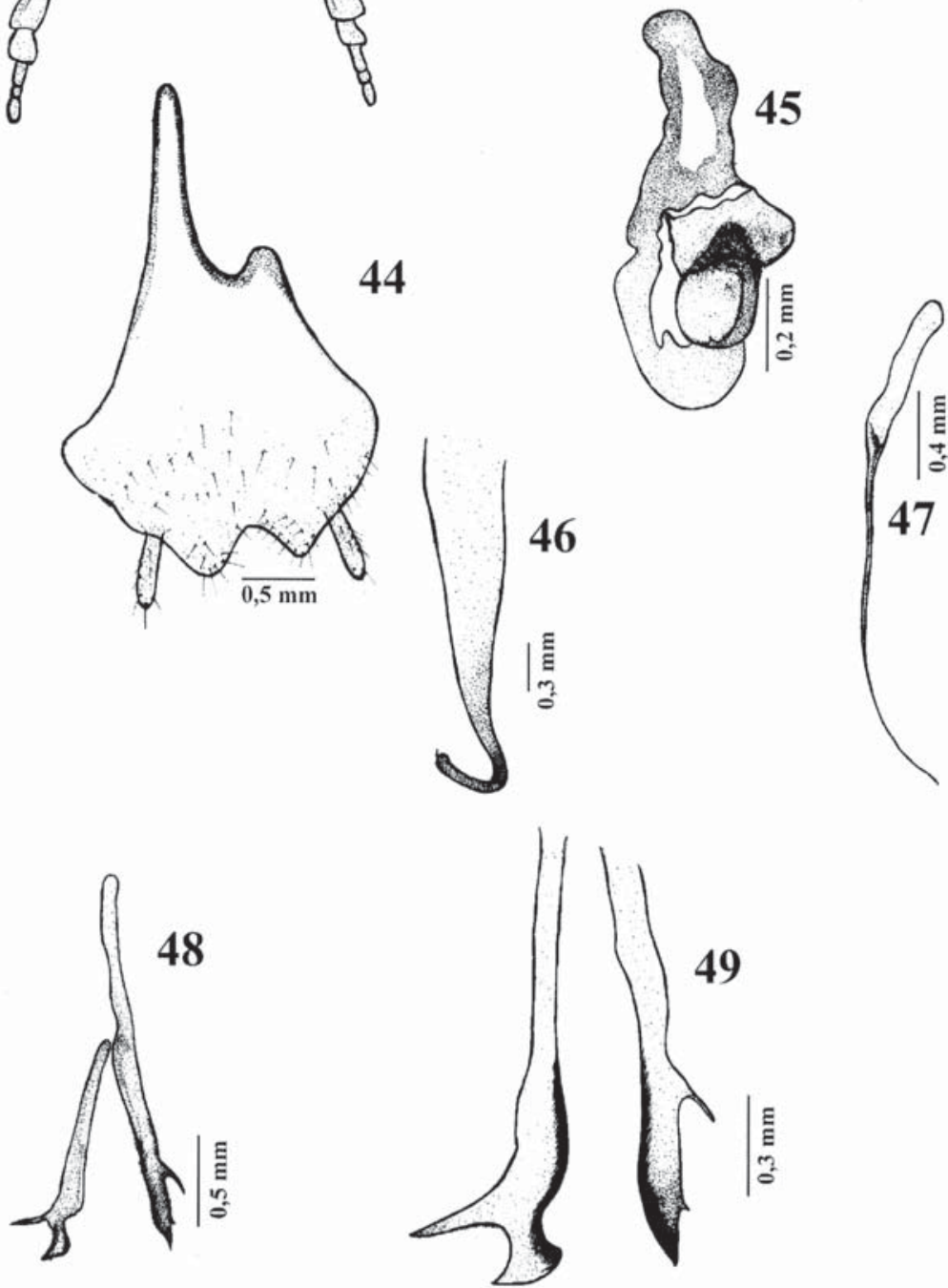

Figs. 42-49. Lophoblatta pellucida (Burmeister, 1838), o. 42, placa supra-anal, dorsal; 43, modificação tergal do abdome, dorsal; 44, placa subgenital, ventral; 45, L1; 46, R2; 47, R3; 48, 49, L2vm. 


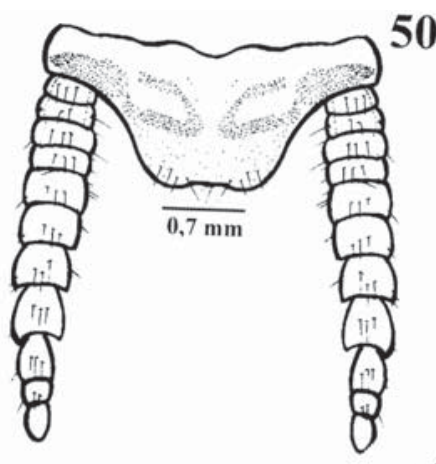

51
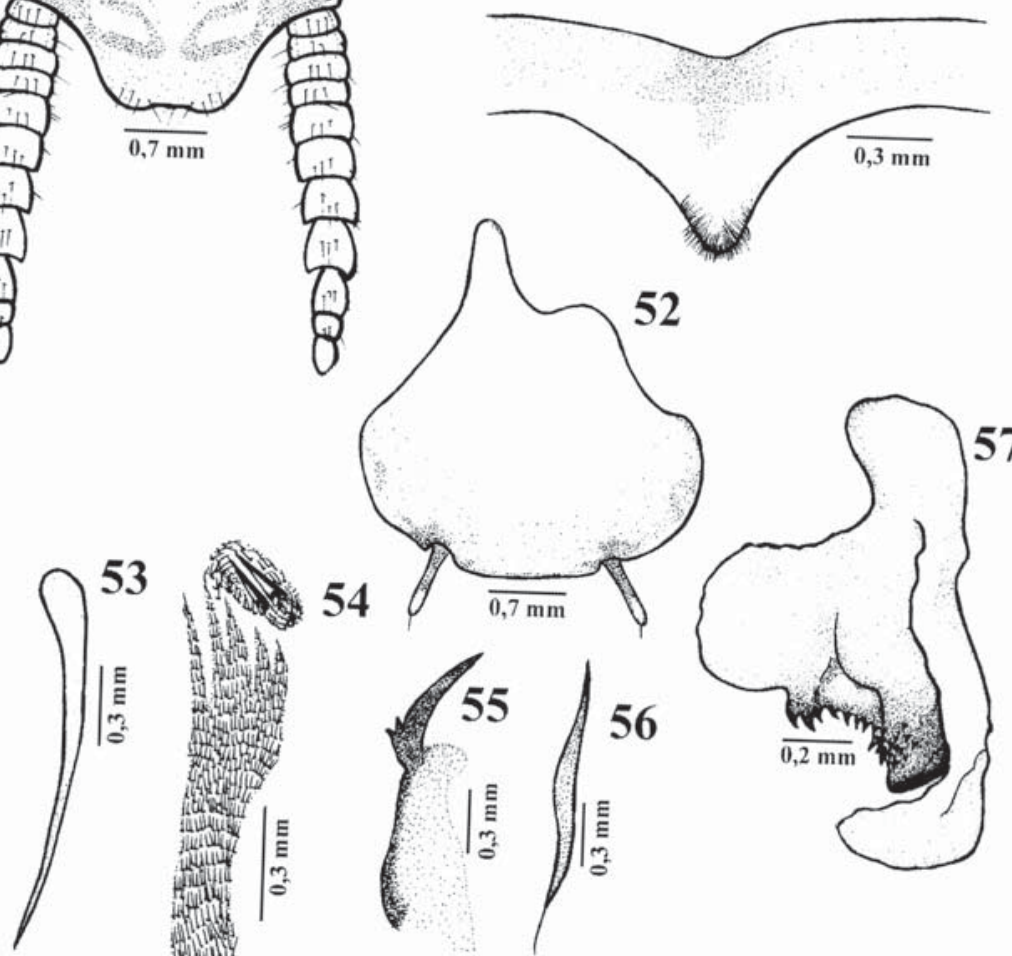

54
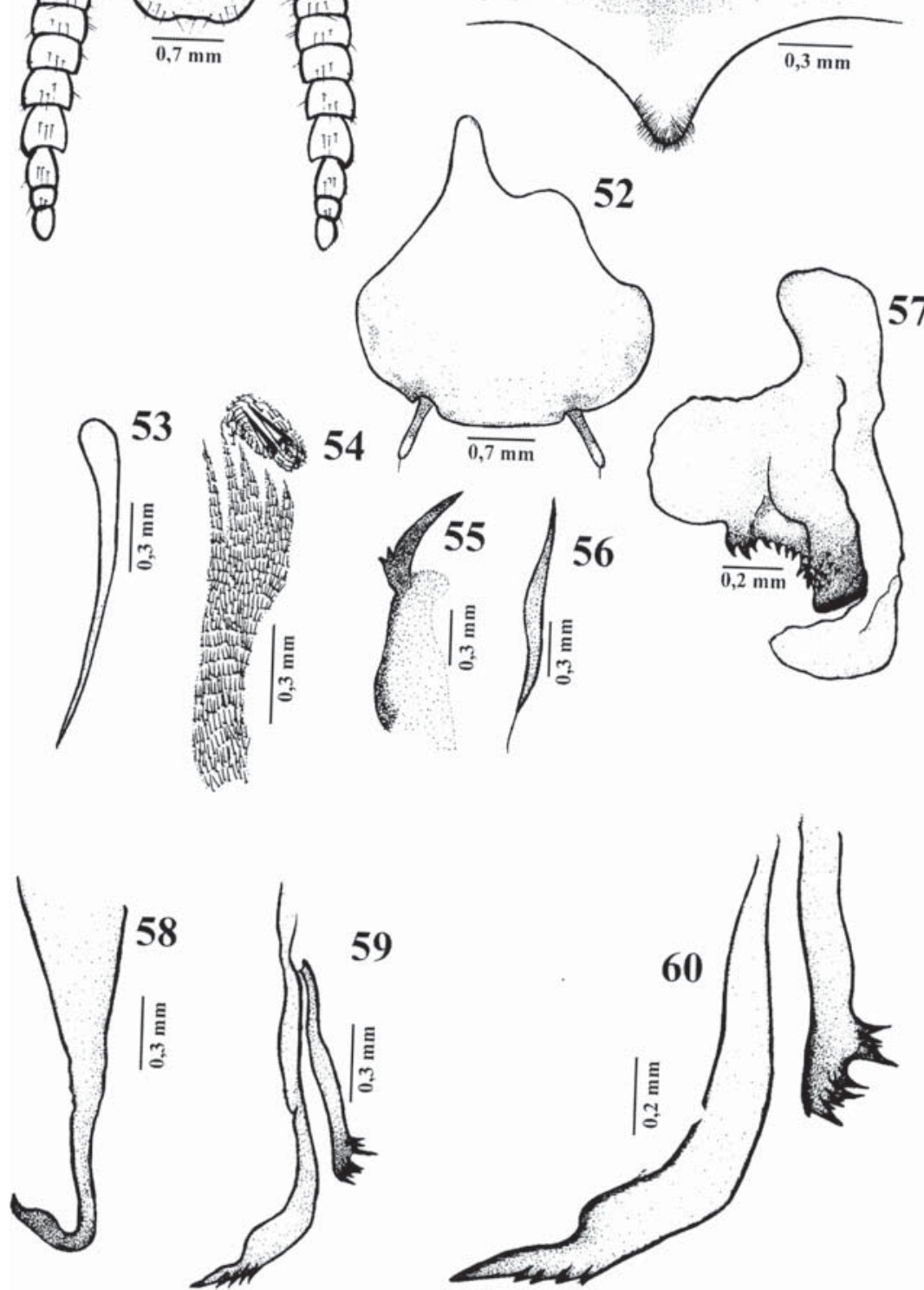

Figs. 50-60. Lophoblatta petropolitana R. e Silva \& Vasconcelos, 1987, đ. 50, placa supra-anal, dorsal; 51, modificação tergal do abdome, dorsal; 52, placa subgenital, ventral; 53, R3; 54, 55, 56, escleritos de R2; 57, L1; 58, R2; 59, 60, L2vm. 

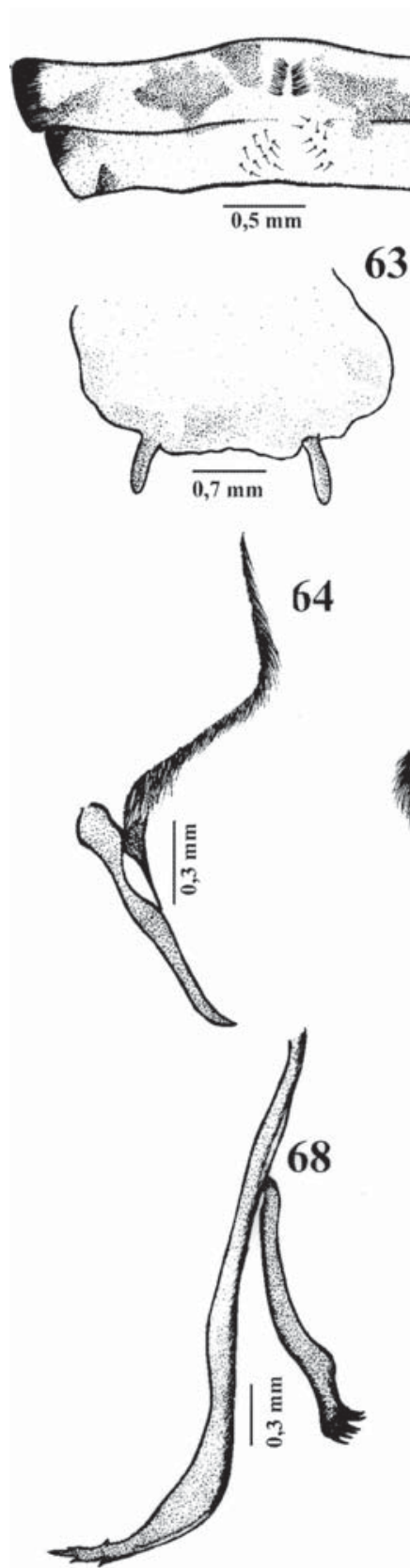

\section{1}

62
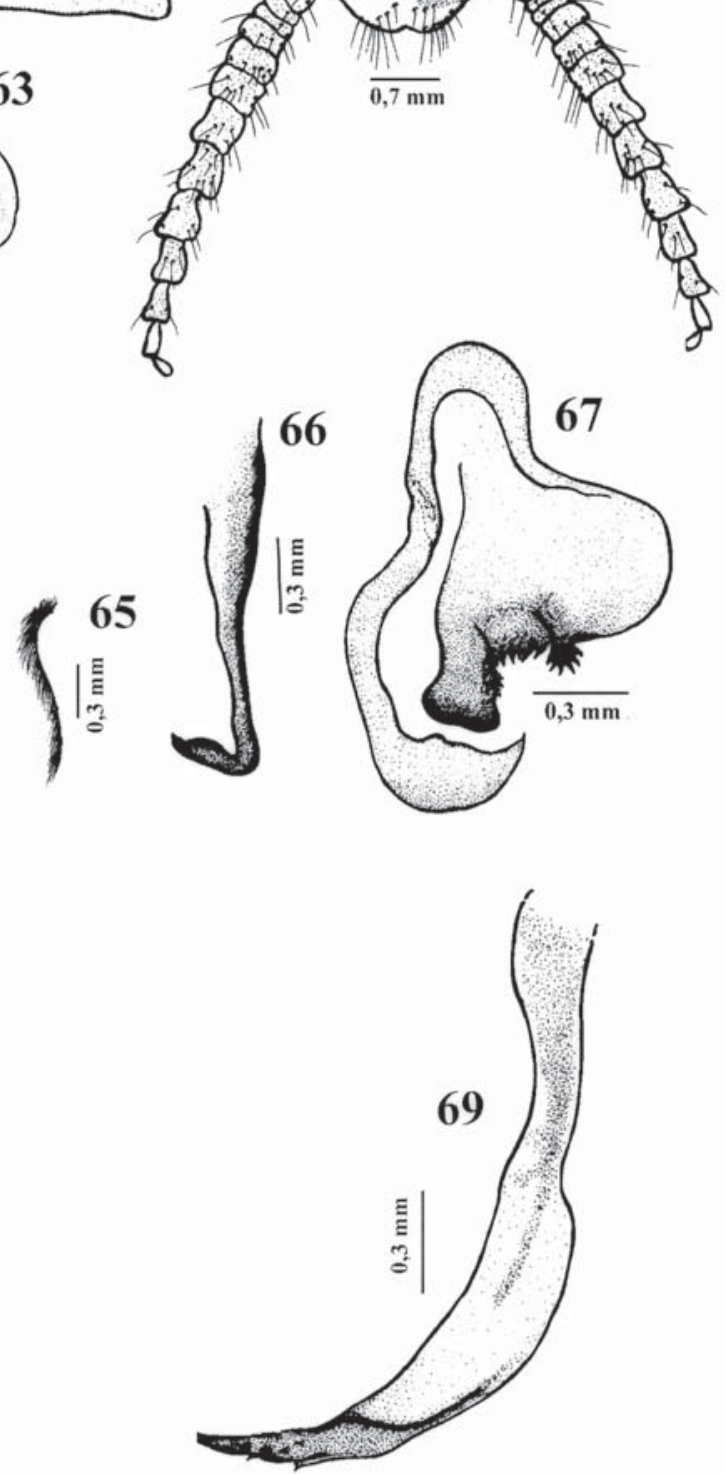

Figs. 61-69. Lophoblatta speerae R. e S.-Albuquerque \& Gurney, 1963, đ. 61, modificação tergal do abdome, dorsal; 62, placa supra-anal, dorsal; 63, placa subgenital, ventral; 64, 65, escleritos de R2; 66, R2; 67, L1; 68, 69, L2vm. 


\section{Lophoblatta speerae Rocha e Silva-Albuquerque \& Gurney, 1963}

(Figs. 61-69)

Lophoblatta speerae R. E S.-Albuquerque \& Gurney, 1963: 525; R. E S.-Albuquerque, 1964a: 26; 1964c: 6; R. e S.-Albuquerque \& Lopes, 1976: 894; 1977: 517; Rocha E Silva, 1982: 14.

Localidade-tipo. Holótipo o, Brasil (São Paulo) (United States National Museum)

Distribuição. Equador, Brasil (Rio de Janeiro, São Paulo). Novas ocorrências: Brasil (Pernambuco, Goiás, Espírito Santo).

§. Abdome com modificação tergal nos segmentos sétimo e oitavo, medianamente com uma pequena fileira simétrica de cílios no sétimo e dispersa no oitavo (fig. 61). Placas supra-anal e subgenital (figs. 62, 63), descritas em R. E S.-AlbuQUERQue \& GURNEY, 1963. L2vm bífido. L2d com ápice acuminado e formações com espinhos subapicais (figs. $68,69)$; ramo lateral levemente estreito na base com uma coroa de espinhos robustos no ápice (fig. 68). L1 com os braços assimétricos: um afilado lateralmente e dilatado no ápice e o outro reduzido; estrutura mediana sem forma definida, apresentando duas projeções: uma formando uma coroa de espinhos e a outra uma expansão apicalmente quadrangular, contendo internamente pequenos espinhos e, entre as duas, uma série de espinhos dispostos linearmente (fig. 67). R2 levemente dilatado próximo ao ápice, que termina em forma de bico de ave (fig. 66). Escleritos do R2 em forma de penacho sinuoso: um esclerotinizado na base e o outro mais afilado (figs. 64, 65).

Material examinado. BRASIL, Pernambuco: Usina Bom Jesus, ơ, $\&$, 17.V.1972, C. Menezes col.; Barreiros (Usina Central Barreiros), ơ, 10.V.1972, Sebastião col.; Distrito Federal: Lago Sul,

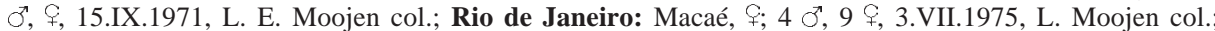
Cabo Frio, ơ, 12.X.1973, N. Santos col., em bromélia Neoregelia cruenta; Arraial do Cabo, ơ, VII.1968, J. Jurberg \& A. L. Perachi col.; Bambuí, \&, 17.VII.1975; Jacarepaguá, ơ, 15.IV.1932, Berla col. (dissecado); Pedra de Itaúna, \&, VII.1965, Santos \& Machado col., em bromélia; Praia de Sernambetiba, \&, V.1984, J.Jurberg et al.col.; Espírito Santo: Parque Sooretama (Cupido),, , 2.III.1948, L. Travassos, Freitas \& H. Travassos col.; São Paulo: Embí-Guaçu, 2 ơ, 6.VII.1982, R. M. Tinoco col., em Vriesia; Caraguatatuba (Reserva Florestal), 40m, ơ, 22.V-1.VI.1962, Exp. Dep. Zool. col.; Ubatuba (Estrada Caraguatatuba), km 222, 2 б, 29.II.1962, em bromélia, Exp. Dep. Zoologia col. (MNRJ).

Discussão. Com base na configuração da placa subgenital do macho e no L2d, o gênero Lophoblatta é dividido em quatro grupos: grupo pellucida (L. brevis, L. pellucida) apresenta a placa subgenital com reentrância mediana muito acentuada entre os estilos; L1 com estrutura mediana pouco esclerotinizada, linguiforme e ápice do L2d afilado e reto, com espinhos diminutos; grupo arawaka (L. arawaka, L. arlei) inclui espécies que possuem a placa subgenital com ápice curvo, sem reentrância mediana entre os estilos; estilos pequenos e afilados e L2d bifurcado; grupo bromelicola ( $L$. bromelicola, $L$. tingua) caracteriza-se por espécies com a placa subgenital reta apicalmente, sem reentrância mediana entre os estilos e ápice do L2d alargado com espinhos em franja; grupo speerae (L. petropolitana, L. speerae) engloba espécies que possuem a placa subgenital com suave reentrância mediana entre os estilos e ápice do L2d afilado, recurvado com pequenos espinhos próximos à extremidade.

Agradecimentos. À Dra. Janira Martins Costa (MNRJ) pelo apoio técnico. Ao Dr. Louis M. Roth (Research Harvard University, Cambridge), pelas sugestões. 


\section{REFERÊNCIAS BIBLIOGRÁFICAS}

Bruijning, C. F. A. 1959. The Blattidae of Surinam. Stud. Fauna Suriname, The Hague, 1:1-103. Brunner, von C. W. 1865. Nouveau système dês Blattaires.Vienna, Société I. R. de Zoologie et de Botanique. 426p.

Burmeister, H. 1838. Blattina. Handb. Ent., Berlin, 2:469-517.

Hebard, M. 1926. The Blattidae of French Guiana. Proc. Acad. nat. Sci. Philad., Philadelphia, 78: $135-244$.

1929. Previously unreported tropical American Blattidae (Orthoptera) in the British Museum. Trans. Am. ent. Soc., Philadelphia, 55:345-388.

Kirby, W. F. 1904. A synonymic catalogue of Orthoptera, Euplexoptera, Cursoria et Gressoria (Forficulidae, Hemimeridae, Blattidae, Mantidae, Phasmidae). London, British Museum London. v. 1, 501p.

Lopes, S. M. \& Oliveira, E. H. 2000a. Material-tipo de Blattaria descrito por Rocha e Silva, depositado na coleção do Museu Nacional-Rio de Janeiro. Publ. Avul. Mus. Nac., Rio de Janeiro, 85:1-24.

2000b. Espécie nova de Eublaberus Hebard, 1919 do Estado de Goiás, Brasil e notas sobre E. marajoara Rocha e Silva-Albuquerque, 1972 (Blaberidae, Blaberinae). Bolm Mus. nac. Rio de J., Nova Série, Zool., Rio de Janeiro, 433:1-5.

McKittrick, F. A. 1964. Evolutionary Studies of Cockroaches. Mem. Cornell Univ. agric. Exp. Stn, Ithaca, 389:1-197.

Princis, K. 1955. Liste der von P. Cornelius Vogl in Venezuela gefangenen Blattarien. Boln Ent. venez., Caracas, $11(1,2): 1-10$.

Princis, K. \& Kevan, D. K. 1955. Cockroaches (Blattariae) from Trinidad B. W. I., with a few records from other parts of the Caribbean. Opusc. ent., Lund, 20:149-169.

Rehn, J. A. G. 1916. The Stanford Expedition to Brazil, 1911. J. C. Branner, Director. Dermaptera and Orthoptera I. Trans. Amer. ent. Soc., Philadelphia, 42:215-308.

1918. On a collection of Orthoptera from the State de Para, Brazil. Proc. Acad. nat sci. Philad., Philadelphia, 70:144-236.

1937. A new species of Blattidae from British Guiana. Ann. Mag. nat. Hist., London, 10 (20):197-203.

1949. Catalogue of the Orthoptera of Venezuela. Part I. Blattidae. Boln Ent. venez., Caracas, 8: $11-19$.

Rocha e Silva-Albuquerque, I. 1964a. Check-list dos Blattaria Brasileiros. Bolm Mus. para. Emilio Goeldi, Nova Série, Zool, Belém, 41:1-37.

1964b. One a collection of cockroaches from Venezuela (Orthoptera, Blattoidea). Bolm Mus. para. Emilio Goeldi, Nova Série, Zool, Belém, 45:1-22.

1964c. Sobre o gênero Lophoblatta Hebard, 1929 com descrição de uma espécie nova (Epilampridae-Blattellinae). Bolm Mus. para. Emilio Goeldi, Nova Série, Zool, Belém, 48:1-8.

1972. Inventário dos Blattaria da Amazônia, com descrição de três espécies novas. Bolm. Mus. para. Emilio Goeldi, Nova Série, Zool., Belém, 76:1-38.

Rocha e Silva-Albuquerque, I. \& Gurney, A. B. 1963. Records and descriptions of cockroaches from southern Brazil (Orthoptera, Blattoidea). Studia. Ent., Petrópolis, 6(1-4):515-536.

Rocha e Silva-Albuquerque, I. \& Lopes, S. M. 1976. Blattaria de Bromélia (Orthoptera). Revta bras. Biol., Rio de J., 36(4):873-901.

1977. Blattaria (Dictyoptera) do Alto da Mosela, Petrópolis, RJ. Revta bras. Biol., Rio de J., 37(3):499-520.

Rocha e Silva, I. 1982. Lista dos Blattodea do município do Rio de Janeiro, RJ, Brasil (Dictyoptera). Bolm Mus. nac. Rio de J., Nova Série, Zool., Rio de Janeiro, 304:1-20.

Rocha e Silva, I. \& VAsconcelos, S. 1987. Nova contribuição ao conhecimento da fauna de Blattaria (Dictyoptera) do Alto da Mosela, Petrópolis, RJ, Brasil, com descrição de três espécies novas.

Bolm Mus. nac. Rio de J., Nova Série, Zool., Rio de Janeiro, 312:1-19.

Roth, L. M. 1968. Oöthecae of the Blattaria. Ann. ent. Soc. Amer., Washington, 61(1):83-111. Shelford, R. 1908. Orthoptera. Fam. Blattidae. Subfam. Phyllodromiinae. Genera Insect., Bruxelles, 73: $1-29$.

Recebido em 16.09.2002; aceito em 06.06.2003 